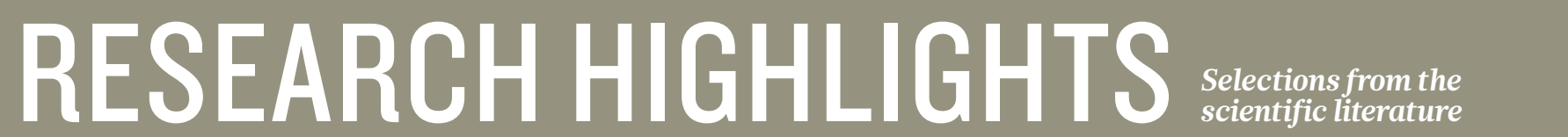

PHYSIOLOGY

\section{How cold triggers fat formation}

In frigid temperatures, mice ramp up the production of heat-generating brown fat by decreasing the levels of a small RNA molecule.

Because brown fat burns energy - unlike its unpopular cousin, white fat - its production is an attractive target for obesity and diabetes therapies. Markus Stoffel at the Swiss Federal Institute of Technology in Zurich and his colleagues found that exposure to cold reduced the expression of microRNA-133 in brown and subcutaneous white fat. Inhibiting miRNA-133 promoted brown-fat formation, whereas forcing miRNA-133 expression switched off brownfat production.

The small RNA acted by directly inhibiting PRDM16, a protein that is central to the production of brown fat from white-fat-cell precursors.

Nature Cell Biol. http://dx.doi. org/10.1038/ncb2612 (2012)

\section{OCEANOGRAPHY}

\section{Thinning ice more fragile and mobile}

The pronounced thinning of Arctic sea ice has made the ice pack more brittle and susceptible to wind drift.

The volume of Arctic sea ice decreased by one-third during 2007-11 compared with the 1979-2006 mean. In a model simulation, Jinlun Zhang at the University of Washington in Seattle and his colleagues demonstrate that the decline in volume substantially reduces the mechanical strength of the ice, thus boosting ice-drift speed and deformation rates.

Forecasts of ice-edge locations will become more challenging as the thinning

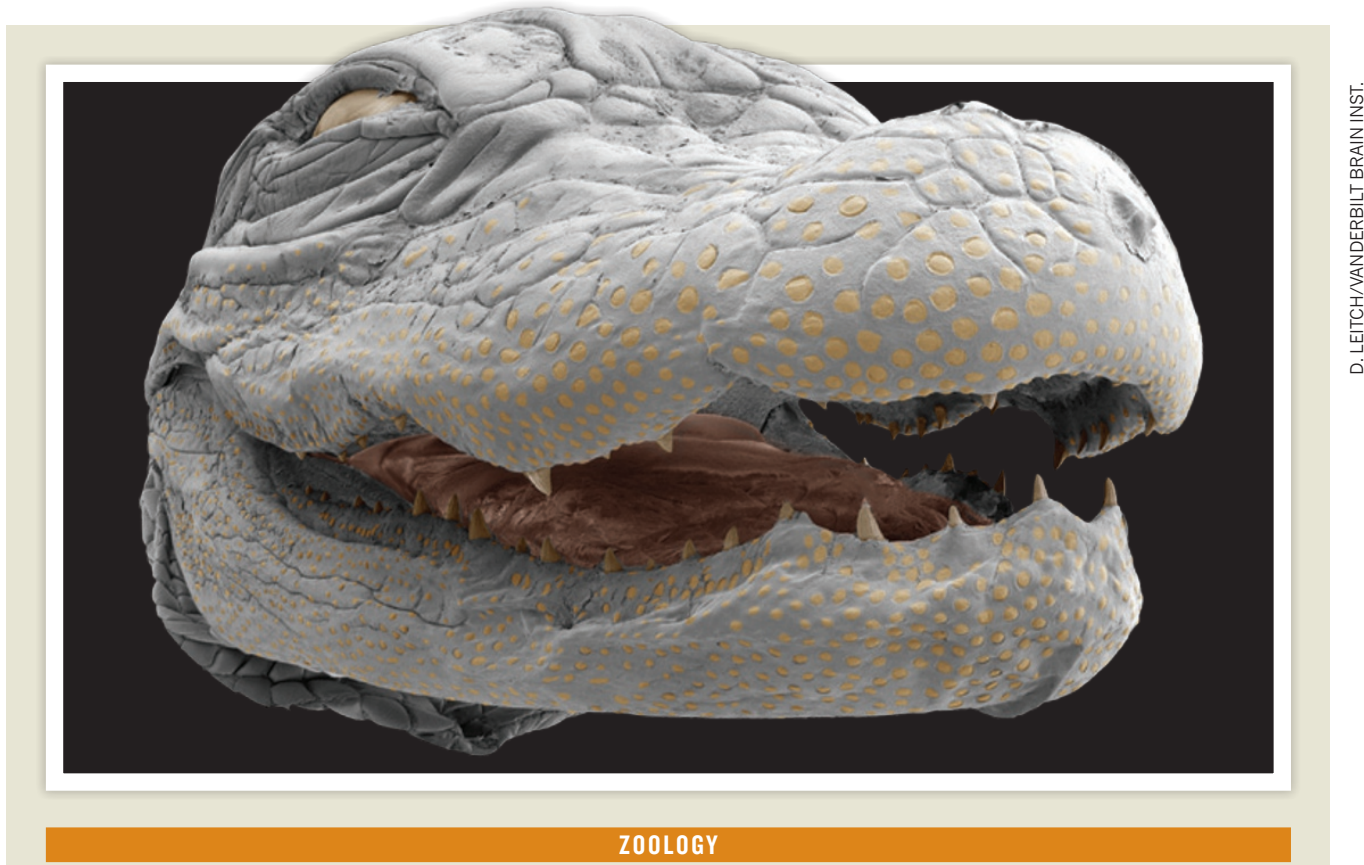

\section{Thick-skinned but sensitive}

Crocodiles and alligators may sense their prey using tiny bumps on their mouths that are highly sensitive to touch.

Kenneth Catania and Duncan Leitch at Vanderbilt University in Nashville, Tennessee, investigated the raised bumps - called integumentary sensory organs - in 18 American alligators (Alligator mississippiensis; hatchling pictured) and 4 Nile crocodiles (Crocodylus niloticus). Confocal microscopy revealed that the bumps (pictured yellow), which are packed most tightly around the teeth and mouth, share similar structures with tactile skin receptors in mammals. The bumps seemed to be insensitive to electrical current or water salinity, but showed nerve responses when stimulated with a range of levels of force - responding to low levels with a sensitivity exceeding that in primate fingertips.

The authors suggest that the animals use the sensitive bumps to locate prey, and to identify food and other items inside their mouths. J. Exp. Biol. 215, 4217-4230 (2012) and weakening of sea ice leads to a state of free drift, the authors note.

Geophys. Res. Lett. http://dx.doi. org/10.1029/2012GL053545 (2012)

\section{NEUROSCIENCE}

\section{Blind reading with sounds}

Blind adults taught to 'read' using sounds that represent letters use the same area of the brain's visual cortex that sighted humans use when reading.

Using a program that 'describes' images in sound,
Amir Amedi at the Hebrew University of Jerusalem in Israel and his team trained eight congenitally blind people to decipher the shapes of letters and objects such as faces and tools. The authors then imaged participants' brains as they listened to sounds associated with letters or other objects. When the volunteers read using the sounds, they activated the same part of the visual cortex - the visual word form area (VWFA) - as sighted controls did when viewing letters.

The work shows that the VWFA is not dependent on visual information alone, and suggests that the visual cortex may be trained to help recognize the environment, even in those who are blind from birth.

Neuron 76, 640-652 (2012)

\section{MATERIALS \\ Counting loops in gels}

Polymer networks, such as rubber and nylon, consist of linked chain-like or branched molecules that almost always contain loops - structural imperfections that weaken a network's connectivity and 